CASE REPORT

\title{
Wernicke's encephalopathy as a complication of gastroparesis after emergency partial antrectomy
}

\author{
N S Ganie, MB ChB; E Janse van Rensburg, MB ChB, MMed (Neurology) \\ Department of Neurology, Faculty of Health Sciences, University of the Free State, Bloemfontein, South Africa
}

Corresponding author: E Janse van Rensburg (vanrense@ufs.ac.za)

Wernicke's encephalopathy is a common complication of malnutrition, alcohol abuse and gastric outlet obstruction. We describe a patient who developed Wernicke's encephalopathy secondary to gastroparesis, with no significant evidence of malnutrition, alcohol abuse, or gastric outlet obstruction.

S Afr Med J 2015;105(2):157. DOI:10.7196/SAMJ.9271

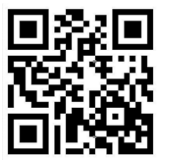

\section{Case report}

A 64-year-old woman with a history of hypertension and peptic ulcer disease was admitted to hospital. She had a 2-month history of vomiting, with difficulty eating and drinking, and felt bloated. These symptoms affected her appetite, resulting in a loss of $10 \mathrm{~kg}$ since symptom onset. Her presentation was further complicated by a recent 1 -week history of visual hallucinations and new-onset generalised tonic-clonic seizures. Her past surgical history revealed an emergency laparotomy for a perforated gastric ulcer for which a partial gastrectomy had been performed. She had a history of severe alcohol abuse, but stopped drinking alcohol $>5$ years ago.

On clinical examination she was haemodynamically stable. She appeared very wasted, with reduced muscle bulk. However, on general examination there was no evidence suggestive of chronic liver disease or pressure sores. Her neurological examination revealed an altered mental state, with a Glasgow coma score of 11/15 (E3M4V4). She responded to commands but spoke little. Cranial nerve examination demonstrated equal pupils, reactive to light bilaterally, with a right conjugate gaze deviation that could not be overcome by the vestibular ocular reflex. An upbeating nystagmus was also noted. The other brainstem reflexes were all intact and there was no meningism. Tonicity in the peripheral limbs was normal, and muscle power was grossly intact globally but difficult to assess. Assessment of deep tendon reflexes revealed an isolated brisk left biceps reflex. All other deep tendon reflexes were normal, with absent clonus. Flexor plantar responses were noted bilaterally. An abdominal examination revealed a soft non-tender abdomen with no palpable organomegaly and no features of chronic alcoholic liver disease. Examination of other bodily systems did not contribute to the final assessment.

A series of haematological, radiological and invasive investigations were performed to investigate the delirium and clinical malnutrition with associated gastrointestinal symptoms. All the haematological investigations were normal, including urea and electrolyte levels, liver function enzymes, albumin levels, white cell count, and haemoglobin level with mean cell volumes. Iron studies and vitamin $B_{12}$ levels were within normal limits, indicating an acute clinical malnutrition without biochemical evidence suggesting otherwise. An endoscopy revealed a small stomach and a healed gastric ulcer with a fibrotic base on the greater curvature. There was also evidence of the anastomosis created by previous surgery. Histological examination of gastric tissue showed no features of dysplasia or malignancy. A water- soluble contrast swallow study was performed, which demonstrated features in keeping with delayed gastric emptying, as contrast media was still notably present in the stomach 5 hours later. In view of the delirium, a computed tomography (CT) scan of the brain was performed, which was reported as normal. Subsequently, a lumbar puncture was done, which was also normal.

As the delirium and new-onset seizures could not be explained, magnetic resonance imaging (MRI) of the brain was performed. Axial T2-weighted images with fluid attenuated inversion recovery (FLAIR) at the level of the basal ganglia showed an increased signal of the fornix and periventricular region around the third ventricle and the medial thalamic nuclei. Images at the level of the midbrain and pons showed an increased signal of the tectal plate. On the sagittal T2-weighted image with FLAIR, an increased signal was noted in the parietal cortex in the post-central gyrus and fornix, mamillary bodies and peri-aqueductal region. These changes were in keeping with Wernicke's encephalopathy.

Treatment with high-dose thiamine was initiated and clinical improvement was noted, reaching a plateau, with the patient being dependent on aftercare for general activities of daily living.

\section{Discussion}

Wernicke's encephalopathy results from a vitamin $B_{1}$ (thiamine) deficiency. Thiamine is a water-soluble vitamin absorbed in the duodenum. Thiamine stores in the body are depleted after approximately 18 days. Therefore, a condition that results in a reduced intake or absorption of thiamine places a patient at risk of developing Wernicke's encephalopathy. Thiamine serves as a co-factor for various enzymes involved with lipid and carbohydrate metabolism. Within a few weeks of thiamine depletion, there is a reduction in the activity of the enzymes involved, the end-result being loss of the osmotic gradients in astrocytes and neuronal tissue, with vasogenic and cytotoxic oedema and subsequent cell necrosis.

The clinical presentation of the typical classic triad of ataxia, confusion and nystagmus is present in $16-38 \%$ of patients. ${ }^{[1]}$ Generally, there is a spectrum of variability of features that comprises the typical triad. Ataxia may present with dizziness and difficulty in walking, with a wide stance and small unsteady steps, leading to complete imbalance and being unable to walk without assistance. Confusion may manifest as a disruption of the patient's mental state, ranging from aggression (if associated with delirium tremens) to apathy and perceptual disturbance, to a global confusional state resulting in 
stupor and coma. Nystagmus may be horizontal or vertical, but may also be associated with ophthalmoplegia. Other abnormalities may include peripheral neuropathy and hypothermia.

Examination of such a patient would include the broad approach taken when investigating delirium of any cause. Haematological investigations are necessary to exclude metabolic or possible infectious causes, and a lumbar puncture is indicated for any confused patient to exclude a meningo-encephalitis. There is insufficient evidence to suggest the use of blood thiamine levels in the diagnosis of Wernicke's encephalopathy. With regard to radiological investigations, a CT scan of the brain will assist in excluding subacute cerebrovascular accidents and mass lesions with surrounding oedema. However, there is evidence that an MRI scan of the brain is the radiological investigation of choice in diagnosing Wernicke's encephalopathy. It has a sensitivity rate of $53 \%$ and a specificity rate of $93 \% .^{[2]}$ Therefore, a diagnosis of Wernicke's encephalopathy can possibly be excluded on an MRI scan. Specific areas of the brain show an increased signal on T2-weighted FLAIR images in Wernicke's encephalopathy. The frequency of involvement of areas from most to least common are as follows: symmetrical lesions of the medial thalami, periventricular regions of the third ventricle, peri-aqueductal areas, mamillary bodies, tectal plate and dorsal medulla. ${ }^{\left[{ }^{3]}\right.}$

Management of Wernicke's encephalopathy involves the replacement of thiamine. There is insufficient evidence from controlled trials pertaining to the dose, frequency, route and duration of thiamine administration. Currently, the Royal College of Physicians proposes that high doses of intravenous thiamine be used, which has been internationally accepted. Initially, intravenous doses of $500 \mathrm{mg} 3$ times daily for 3 days should be administered, followed by
$250 \mathrm{mg}$ daily, until there is resolution of symptoms or plateauing of improvement. Thereafter, continuous supplementation is required for patients who remain at risk. ${ }^{[4]}$ The initial intravenous route for thiamine correction is based on the poor bioavailability of oral thiamine, ranging from $3.7 \%$ to $5.3 \%{ }^{[5]}$

In a systematic review of Wernicke's encephalopathy after bariatric surgery ${ }^{[6]}$ it was shown that any gastrointestinal procedure resulting in weight loss can predispose a patient to thiamine deficiency. Vomiting is a complication of gastrointestinal procedures and can also be caused by upper-intestinal obstruction. In our patient, in view of the previous emergency laparotomy that she had undergone for a perforated peptic ulcer, we hypothesise that a complication of that surgery was the partial denervation of the smooth muscle of the stomach. As a result, even though there was no gastric outlet obstruction, there was gastroparesis, resulting in severe persistent vomiting in an already malnourished patient at risk of the development of Wernicke's encephalopathy.

\section{References}

1. Sechi G1, Serra A. Wernicke's encephalopathy: New clinical settings and recent advances in diagnosis and management. Lancet Neurol 2007;6(5):442-455. [http://dx.doi.org/10.1016/S1474$4422(07) 70104-7$

2. Antunez E1, Estruch R, Cardenal C, et al. Usefulness of CT and MR imaging in the diagnosis of acute Wernicke's encephalopathy. AJR Am J Roentgenol 1998;171(4):1131-1137. [http://dx.doi.org/10.2214/ ajr.171.4.9763009]

3. Zuccoli G1, Santa Cruz D, Bertolini M, et al. MR imaging findings in 56 patients with Wernicke encephalopathy: Nonalcoholics may differ from alcoholics. AJNR Am J Neuroradiol 2009;30(1):171-176. [http://dx.doi.org/10.3174/ajnr.A1280]

4. Thomson AD, Cook CC, Touquet R, et al., Royal College of Physicians, London. The Royal College of Physicians report on alcohol: Guidelines for managing Wernicke's encephalopathy in the accident and emergency department. Alcohol 2002;37(6):513-521. [http://dx.doi.org/10.1093/alcalc/37.6.513]

5. Weber W, Kewitz H. Determination of thiamine in human plasma and its pharmacokinetics. Eur $J$ Clin Pharmacol 1985;28(2):213-219. [http://dx.doi.org/10.1007/BF00609694]

6. Aasheim ET. Wernicke encephalopathy after bariatric surgery: A systematic review. Ann Surg 2008:248(5):714-720. 